\title{
An energy-efficient full-duplex MAC protocol for distributed wireless networks.
}

\author{
AL-KADRI, M.O., AIJAZ, A., NALLANATHAN, A.
}

2016

(C) 2016 IEEE. Personal use of this material is permitted. Permission from IEEE must be obtained for all other uses, in any current or future media, including reprinting/republishing this material for advertising or promotional purposes, creating new collective works, for resale or redistribution to servers or lists, or reuse of any copyrighted component of this work in other works. 


\title{
An Energy-Efficient Full-Duplex MAC Protocol for Distributed Wireless Networks
}

\author{
M. Omar Al-Kadri, Adnan Aijaz*, Member, IEEE, and Arumugam Nallanathan, Senior Member, IEEE
}

\begin{abstract}
In this paper, we present an energy-efficient medium access control (MAC) protocol for distributed full-duplex (FD) wireless network, termed as Energy-FDM. The key aspects of the Energy-FDM include energy-efficiency, co-existence of distinct types of FD links, throughput improvement, and backward comparability with conventional half-duplex (HD) nodes. Performance evaluation demonstrates the effectiveness of proposed protocol as a viable solution for full-duplex wireless networks.
\end{abstract}

Index Terms-full-duplex, MAC protocol, energy-efficient.

\section{INTRODUCTION}

$\mathbf{E}$ XISTING efforts towards full-duplex (FD) wireless communications have mainly focussed on investigating the Physical (PHY) layer aspects [1]. However, novel solutions and protocol enhancements are needed at higher layers to achieve the true benefits of FD technology.

In FD distributed wireless networks, design of medium access control (MAC) layer becomes particularly challenging [2]. Some recent studies have proposed different MAC protocols for FD distributed wireless networks. In [3], the authors proposed an FD MAC protocol specifically designed for bidirectional links. In [4], ContraFlow MAC protocol has been presented. To avoid collisions and to solve the hidden node problem, the primary receiver in ContraFlow sends a busy tone even if it has no data to transmit, which is inherently inefficient in terms of power/energy consumption. Besides, ContraFlow does not exploit the uni-directional links. In [5], the authors propose a distributed MAC protocol that considers uni-directional links. However, a new one bit transmission flag (TF) has been introduced to identify the FD opportunity, which makes the protocol backward incompatible with conventional half-duplex (HD) nodes. In [6], a MAC protocol has been proposed that accounts for uni-directional links and addresses the hidden node problem without the use of busy tone. The above mentioned MAC protocols are based on carrier sense multiple access (CSMA). Energy efficiency has been rarely considered in existing protocols. All aforementioned protocols use maximum transmission power for control and data packets, which is not energy-efficient.

Against this background, the main contribution of this paper is to extend [6] for achieving energy efficiency, while particularly accounting for the peculiarities of FD environments such as bi-directional and uni-directional links (explained in Section

M. O. Al-Kadri and A. Nallanathan are with the Centre for Telecommunications Research, King's College London, London, UK.

A. Aijaz is now with Toshiba Research Europe Ltd., Bristol, UK.

* This work was done while the author was working at the Centre for Telecommunications Research, King's College London, London, UK.

E-mail: mhd_omar.alkadri@kcl.ac.uk
II). The protocol operation of [6] is adapted for the proposed energy saving technique. The proposed protocol, termed as Energy-FDM, particularly focuses on reducing the transmission power of data and acknowledgement (ACK) packets to achieve energy efficiency. It also ensures operability of both bi-directional and uni-directional links, maintains backwards compatibility with co-existing HD nodes, and achieves high throughput by using FD while addressing the hidden node problem. The proposed protocol is analysed through a stochastic geometry based approach for accurate and realistic performance evaluation.

\section{SYSTEM MODEL}

We consider a Poisson distributed wireless network comprising both FD and HD nodes. For FD operation, we adopt a PHY layer model from [7] wherein, each node is equipped with a single shared antenna along with the proposed selfinterference (SI) cancellation mechanism therein. We consider two types of FD wireless links. Bi-directional links (Bi-Links), where node A transmits to node B in the first transmission (FT) and node $\mathrm{B}$ transmits to node $\mathrm{A}$ in the second transmission (ST), with both FT and ST occurring simultaneously. In this case, both nodes suffer from SI and apply the necessary SI cancellation mechanism. Uni-directional links (Uni-Links), where node A transmits to B (FT) and B transmits to another node C (ST). In this case, only node B experiences SI.

Further, we define the following ranges for each node in the network, which are also illustrated in Fig. 1. 1) Transmission Range: In which any residing node can successfully decode the transmitted packets by a sender. 2) Carrier Sensing Range: In which residing nodes can sense the sender's transmission. 3) Carrier Sensing Zone: In which any residing node cannot successfully decode the transmitted packets by a sender node, and consequently sets its Network Allocation Vector (NAV) to Extended Inter-Frame Spacing (EIFS) ${ }^{1}$. The carrier sensing zone excludes the transmission range.

\section{ENERGY-FDM PROTOCOL}

In our underlying system model, three distinct types of communications can occur: (a) FD bi-directional communication via Bi-links, (b) FD uni-directional communication via Unilinks, and (c) conventional HD communication. Consider that node $\mathrm{A}$ has data to send to node $\mathrm{B}$. It takes a random back-off (BO) if the channel is sensed as busy. After the expiry of BO timer, if the channel is sensed as idle, it starts the transmission

\footnotetext{
${ }^{1}$ This is done in order to provide the intended receiver with an opportunity to return an acknowledgement (ACK) without interference.
} 


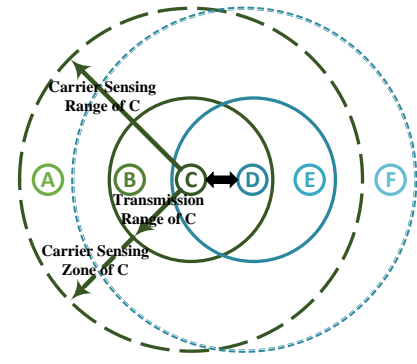

Fig. 1: Range definitions in the underlying system model.

by sending a request-to-send (RTS) packet to node B with maximum power $\left(P_{\max }\right)$, in order to capture the channel and to make other nodes aware of an ongoing transmission. Note that this is the FT from node A which includes the source and destination addresses as well as the length of the transmission.

\section{A. FD Bi-directional Communication}

In case of FD bi-directional communication between nodes A and B, after receiving the RTS packet from A, node B waits for short inter-frame space (SIFS) duration before sending an FD clear-to-send (FD-CTS) packet A. The FD-CTS packet includes the source and destination addresses along with the transmission durations of both FT and ST. Note that FD-CTS is also transmitted using $P_{\max }$ to capture the channel for ST. Node B also calculates $P_{\text {min }}$, which is defined as the minimum transmit power for successful data transmission such that

$$
P_{\text {min }}=\frac{P_{\max }}{P_{r x}} \times R_{x}^{\text {thresh }} \times c,
$$

where $P_{r x}$ is the received power, $R_{x}^{\text {thresh }}$ is the minimum necessary received signal strength and $c$ is a constant as [8].

Once node A receives the FD-CTS, it calculates $P_{\min }$ as well, and responds with another FD-CTS for synchronization with node B. After B receives FD-CTS from A, data transmission starts using $P_{\min }$ with periodical increase to $P_{\max }$, so that nodes in the sensing zone, which cannot decode the transmission and set their NAV to EIFS duration can sense the transmission. Note that the period between two successive power increase intervals must be less than the EIFS duration. According to the IEEE 802.11 standard [9], $15 \mu \mathrm{s}$ is suitable for carrier sensing, and $2 \mu \mathrm{s}$ is adequate to increase/decrease the power level from/to $10 \%$ to/from $90 \%$. Therefore, a duration of $20 \mu \mathrm{s}$ is deemed adequate for transition of power level from $P_{\min }$ to $P_{\max }$ and vice versa, as shown in Fig. 2. Since EIFS is set to $364 \mu s$ in IEEE 802.11 standard, a node in Energy-FDM will transmit at $P_{\max }$ every $340 \mu \mathrm{s}$ for a duration of $20 \mu \mathrm{s}$, and the cumulative transmission duration is less than the EIFS duration. Further, since the durations of FT and ST are known, the transmission will last for the longer duration, after which both nodes A and B will send an ACK. Note that the protocol operation inherently accounts for the necessary SIFS duration as shown in Fig. 2

\section{B. FD Uni-directional Communication}

The protocol operation in this case is illustrated in Fig. 2. If node $\mathrm{B}$ has packets to send to another node $\mathrm{C}$, it waits for
SIFS and then sends FD-CTS to both A and C using $P_{\max }$, after which it calculates $P_{\min }$ using the received power from $A$. Next, node A calculates $P_{\min }$ and waits for a duration of 2 SIFS and an FD-CTS before initiating data transmission. When node $\mathrm{C}$ receives FD-CTS from $\mathrm{B}$, it calculates $P_{\min }$ and waits for SIFS duration before sending FD-CTS back to node B. After that node B calculates $P_{\min }$ using the received power from node $\mathrm{C}$ and compares it with the previously calculated $P_{\min }$ (from node A). Node B will use the higher $P_{\text {min }}$ in order to maintain both FT and ST connections. The data transmission lasts for the longer duration of either FT or ST using $P_{\min }$ with periodic increase to $P_{\max }$, as described earlier. After the completion of data transmission, node $\mathrm{C}$ sends ACK to node B, and B sends ACK to node A.

\section{HD Communication}

It is important to maintain backward compatibility with conventional HD nodes. If node B is an HD node, or does not have packets to send, it waits for SIFS duration and sends back a normal CTS to node A. The communication will proceed as HD based on the standard 802.11 DCF protocol [10].

\section{Solving Hidden Nodes Problem}

Refering to Fig. 1, consider that nodes C and D constitute a sender-receiver pair in HD mode. Node F, which resides in the carrier sensing range of $\mathrm{D}$ but not of node $\mathrm{C}$, may act as a hidden node. In bi-directional transmission, both nodes transmit and receive simultaneously, and therefore the hidden node issue is implicitly resolved due to the FD nature of this transmission. In uni-directional transmission, hidden nodes may affect the receiver of ST. That is why Energy-FDM adopts RTS-CTS mechanism, and by sending FD-CTS using $P_{\max }$, it ensures that nodes in the carrier sensing range of ST are aware of an ongoing transmission.

\section{PROTOCOL ANALYSis}

\section{A. Spatial Throughput Analysis}

We assume that nodes are distributed in an Euclidean plane $\mathbb{R}^{2}$ according to a homogeneous Poisson Point Process $\Phi$ with intensity $\lambda$. Node A located at $x$ correctly receives and decodes packets transmitted from node B located at $y$ if the received $\operatorname{SINR}(\gamma)$ is higher than a threshold $\beta$ i.e.,

$$
\gamma(A)=\frac{P_{r x}}{N_{0}+I_{x}}>\beta,
$$

where $N_{0}$ is the noise power, and $I_{x}$ is the cumulative interference of all other transmitting nodes in the contention domain of node A. Similar to [11], we adopt the modified Matérn model for MAC layer contention which is suitable to describe the synchronized and slotted version of CSMA and hence complies with our protocol. In this model, each point $x$ of $\Phi$ is attributed an independent mark $t_{x}$ uniformly distributed in $[0,1]$. A node transmits if it does not sense the activity of any other node having smaller mark. The subset of neighboring nodes of $x$ transmitting concurrently is given by

$$
\mathcal{N}(x)=\left\{x \in \Phi: t_{x}<t_{y}, \forall y: P_{r x}>\beta\right\} .
$$




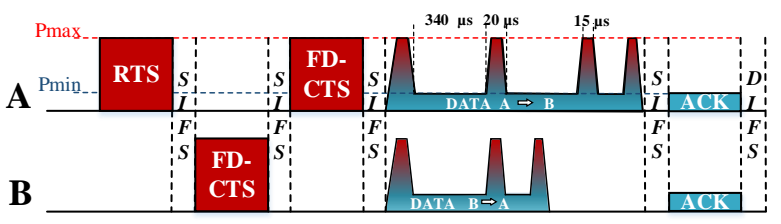

Bi-directional transmission

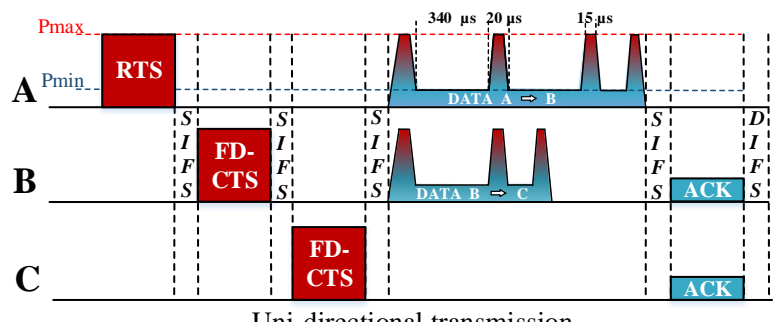

Uni-directional transmission

Fig. 2: Example of the bi-directional and uni-directional transmission in Energy-FDM using 1 Mbps bandwidth.

The received power is given by $P_{r x}=P \cdot L(x, y) \cdot F(x, y)$, where $P$ is the transmitted power (either $P_{\max }$ or $P_{\min }$ ), $L(x, y)$ is the the path loss component, and $F(x, y)$ is a random variable accounting for the fading and shadowing. We adopt the notion of average spatial throughput from [11] and calculate for our scenario which depends on: (i) distance distribution between a pair of nodes, (ii) set of simultaneously transmitting nodes, and (iii) probability of successful transmission, and is given by

$$
\tau_{F D}=\int_{0}^{\infty} \int_{0}^{\infty}\left(\alpha \mathbb{P}_{B i}+(1-\alpha) \mathbb{P}_{U n i}\right) f_{D}(r, n) \mathrm{d} r \mathrm{~d} n
$$

where $\alpha$ is the fraction of Bi-links, $r$ is the distance between sender and receiver nodes, $n=|\mathcal{N}(\cdot)|, \mathbb{P}_{B i}$ and $\mathbb{P}_{U n i}$ respectively denote the probabilities of successful bi-directional and uni-directional transmissions, and $f_{D}(r, n)$ is the probability density function of the distance between the receiver and the neighbours in the contention domain, given by [12]

$$
f_{D}(r, n)=\frac{\left(\pi \lambda r^{2}\right)^{n-1}}{(n-1) !} e^{-\pi \lambda r^{2}} .
$$

Following the stochastic analysis of random networks in Section 3.2 of [13], we define the probability of successful transmission in our case as follows:

$$
\begin{aligned}
\mathbb{P} & =\left(\frac{\left(\mathcal{L}_{I_{1}}\right)^{n-2}}{\left(1+\Omega\left(\beta x^{\ell_{1}}, x\right)+B\left(1-\frac{2}{\ell_{1}}, 1+\frac{2}{\ell_{1}}\right) \beta^{\frac{2}{\ell_{1}}}\right)}\right) \\
& \times\left(\frac{\left(\mathcal{L}_{I_{2}}\right)^{n-2}}{\left(1+\Omega\left(\beta y^{\ell_{2}}, y\right)+B\left(1-\frac{2}{\ell_{2}}, 1+\frac{2}{\ell_{2}}\right) \beta^{\frac{2}{\ell_{2}}}\right)}\right)
\end{aligned}
$$

where $\mathcal{L}_{I_{1}}$ and $\mathcal{L}_{I_{2}}$ respectively denote the Laplace transforms of the interfering neighbours of FT and ST receivers, $\ell_{1}$ and $\ell_{2}$ respectively denote the path loss exponent between pair of FT and ST nodes, $B(\cdot, \cdot)$ is the Beta function, and $\Omega(s, X)$ is a function defined as

$\Omega(s, X)=\frac{\mathcal{F}\left(1,1,1-\frac{2}{\ell}, \frac{s X^{-\ell}}{1+s X^{-\ell}}\right)}{1+s X^{-\ell}}-B\left(1-\frac{2}{\ell}, 1+\frac{2}{\ell}\right) s^{\frac{2}{\ell}} X^{-2}-1$

where $\mathcal{F}(\cdot, \cdot, \cdot, \cdot)$ is the Gaussian Hypergeometric function. Note that (6) is valid for both Bi-links and Uni-links.

\section{B. Energy Analysis}

Owing to the presence of SI, it is important to consider FD efficiency [6] for a FD node, defined as the ratio of the effective received packet payload to the sent packet payload, and given by $\epsilon=\frac{\int_{0}^{\infty} \log _{2}(1+\kappa \gamma) f(\gamma) d \gamma}{\int_{0}^{\infty} \log _{2}(1+\gamma) f(\gamma) d \gamma}$, where $\gamma$ is the instantaneous received SINR at the FD node, $f(\gamma)$ is the probability density function of the channel and $\kappa \in[0,1]$ is the SI cancellation coefficient. When $\kappa \rightarrow 0$ the SI causes large interference on the FD transmission, whereas when $\kappa \rightarrow 1$ the SI causes no interference. In bi-directional transmission, the FD efficiency is taken into account for both nodes as both operate in FD mode, while in uni-directional transmission, the efficiency is considered only for the FT receiver node as it is the only FD node. The effective packet payload $\delta_{F D}$ (in bits) is given by

$$
\delta_{F D}=\left\{\begin{array}{ll}
\delta_{B i} & =\epsilon\left(\delta_{F T}+\delta_{S T}\right) \\
\delta_{U n i} & =\epsilon \delta_{F T}+\delta_{S T}
\end{array},\right.
$$

where $\delta_{B i}$ and $\delta_{U n i}$ are the effective packet loads of the Bilinks and Uni-links respectively, $\delta_{F T}$ and $\delta_{S T}$ are the packet payload for the FT and ST respectively. For the sake of comparison, we define the effective packet payload of a HD link as $\delta_{H D}=\frac{\delta_{F T}+\delta_{S T}}{2}$.

Let, $E_{B i}=E_{A}^{B i}+E_{B}^{B i}$ denote the average energy consumed during a successful FD bi-directional transmission, where $E_{A}$ and $E_{B}$ denote the energy consumed by nodes $\mathrm{A}$ and $\mathrm{B}$ respectively, such that

$$
\begin{aligned}
E_{A}^{B i}= & T_{F D C T S} \cdot P_{o n}+\left(T_{\delta_{F T}}-T_{I F T}+T_{A C K}\right) P_{\min } \\
& +\left(T_{R T S}+T_{F D C T S}+T_{I F T}\right) P_{\max } \\
E_{B}^{B i}= & \left(T_{R T S}+T_{F D C T S}\right) P_{o n}+\left(T_{F D C T S}+T_{I S T}\right) P_{\max } \\
& +\left(T_{\delta_{S T}}-T_{I S T}+T_{A C K}\right) P_{\min }
\end{aligned}
$$

where $T_{R T S}, T_{F D C T S}, T_{H}$ and $T_{A C K}$ denote the duration of RTS, FD-CTS, header (of both MAC and PHY) and ACK frames respectively, SIFS and DIFS denote the short and DCF inter-frame space duration (defined in the 802.11 DCF standard) respectively, $T_{\delta_{F T}}$ and $T_{\delta_{S T}}$ denote the duration of effective payload for FT and ST transmissions respectively, $P_{o n}$ is the power consumed during the receive mode, and $T_{I F T}$ and $T_{I S T}$ are the durations of power increase to $P_{\max }$ for the FT and ST respectively such that $T_{I F T}=20 \cdot\left(\frac{\delta_{F T}}{E I F S}+1\right)$ and $T_{I S T}=20 \cdot\left(\frac{\delta_{S T}}{E I F S}+1\right)$. Note that the transmission duration (in $\mu \mathrm{s}$ ) for $\mathrm{c}$ bits is calculated as $T_{c}=c / \mathcal{B}$, where $\mathcal{B}$ is the bandwidth.

Similarly, let $E_{U n i}=E_{A}^{U n i}+E_{B}^{U n i}+E_{C}^{U n i}$ denote the average energy consumed during a successful FD uni-directional 


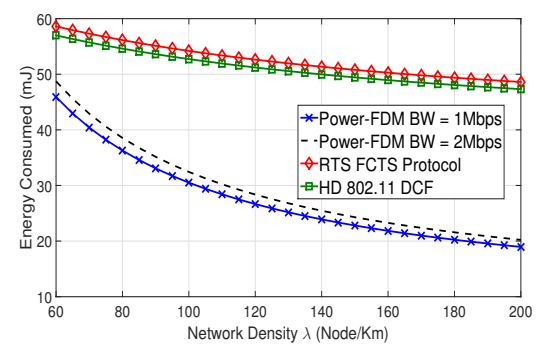

(a)

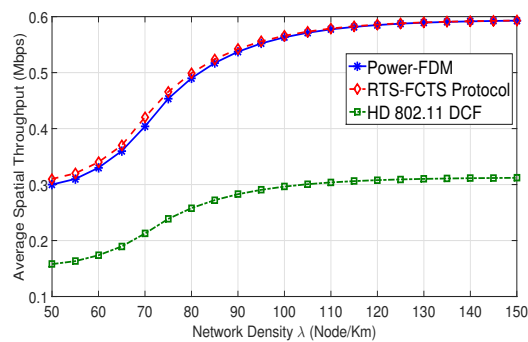

(b)

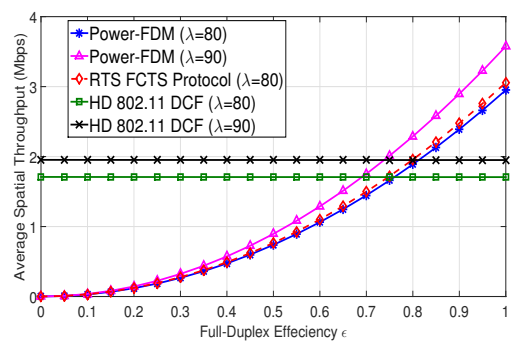

(c)

Fig. 3: Performance evaluation of Energy-FDM, (a) energy consumption against network density, (b) average spatial throughput against network density, (c) average spatial throughput against FD efficiency (BW denotes the bandwidth).

transmission such that

$$
\begin{aligned}
E_{A}^{U n i}= & \left(T_{R T S}+T_{I F T}\right) P_{\max }+\left(2 T_{F D C T S}+T_{A C K}\right) P_{o n} \\
& +\left(T_{\delta_{F T}}-T_{I F T}\right) P_{\min }, \\
E_{B}^{U n i}= & \left(T_{R T S}+T_{F D C T S}\right) P_{r x}+\left(T_{F D C T S}+T_{I S T}\right) P_{\max } \\
& +\left(T_{\delta_{S T}}-T_{I S T}+T_{A C K}\right) P_{\min } \\
E_{C}^{U n i}= & \left(T_{R T S}+T_{F D C T S}+T_{\delta_{S T}}\right) P_{o n} \\
& +T_{F D C T S} P_{\max }+T_{A C K} P_{\min } .
\end{aligned}
$$

Therefore, the total energy consumption is given by $E=$ $\alpha E_{B i}+(1-\alpha) E_{U n i}$.

\section{NumERicAl RESUlts}

We assume Poisson distributed nodes in an area of $1500 \mathrm{~m}^{2}$ and vary the density of nodes. Further, we assume 1 Mbps bandwidth with maximum transmit power of $24 \mathrm{dBm}$. The frame size (in bits) of different packets is set to 277 for RTS, 528 for FD-CTS, 240 for CTS, 240 for ACK, 128 for PHY header, 272 for MAC header, and 8184 for payload. Different inter-frame spacing durations (in $\mu s$ ) are set to as, 28 for SIFS, 128 for DIFS, 364 for EIFS. The slot duration is set to as 50 $\mu s$. Lastly, we assume $\alpha=0.5$. We adopt the FD protocol RTS-FCTS [6] as the baseline as it uses similar control signals with maximum power. We also compare the performance with the standard IEEE 802.11 (HD) DCF protocol [10].

Fig. 3a shows the energy consumption for different protocols. We note that the energy consumption reduces with the network density. This is because of overall reduced transmission power owing to shorter transmission links. Further, Energy-FDM outperforms the baselines protocols by consuming on average up to $47.2 \%$ and $44.8 \%$ lower energy compared to [6] and [10], respectively. The energy consumption increases with increase in bandwidth as the transmission duration of different messages as well as the inter-frame spacing durations decrease, as a result of which, the power must be increased more frequently during data transmission.

Fig. $3 \mathrm{~b}$ shows the average spatial throughput performance for different protocols. We note that despite reducing the transmission power, Energy-FDM achieves similar throughput performance as [6] which transmits at maximum power. The throughput initially increases in low network density, where collision effects are not significant. After reaching the maximum value, it stops due to network saturation.
In FD networks, performance is heavily dependent on the SI cancellation. As shown by results in Fig. 3c, the average spacial throughput increases when the FD efficiency $(\epsilon)$ increases. We note that it drops to the level of HD system when $\epsilon$ drops below 0.75 . Therefore, SI must be dealt with properly to achieve the gain of FD technology at higher layers.

\section{CONCLUDING REMARKS}

We have proposed Energy-FDM, which is an energyefficient FD MAC protocol for distributed wireless networks. Energy-FDM achieves higher energy-efficiency without compromising the overall effective throughput. Moreover, it supports both Bi-links and Uni-links and is inherently backward compatible. Performance evaluation shows the achievable gains of Energy-FDM. Results also demonstrate that higher layer gains of FD technology heavily rely on SI cancellation mechanisms at the PHY layer.

\section{REFERENCES}

[1] D. Kim, H. Lee, and D. Hong, "A Survey of In-band Full-duplex Transmission: From the Perspective of PHY and MAC Layers," IEEE Commun. Surveys Tuts., no. 99, 2015.

[2] X. Xie and X. Zhang, "Does Full-Duplex Double The Capacity of Wireless Networks?" in IEEE INFOCOM, 2014, pp. 253-261.

[3] S. Kim and W. E. Stark, "On The Performance of Full Duplex Wireless Networks," in IEEE CISS, 2013, pp. 1-6.

[4] N. Singh et al., "Efficient and Fair MAC for Wireless Networks With Self-Interference Cancellation," in IEEE WiOpt, 2011, pp. 94-101.

[5] S. Goyal et al., "A Distributed MAC Protocol for Full Duplex Radio," in IEEE REC ASILOMAR C, 2013, pp. 788-792.

[6] W. Cheng, X. Zhang, and H. Zhang, "RTS/FCTS Mechanism Based FullDuplex MAC Protocol for Wireless Networks," in IEEE GLOBECOM 2013, pp. 5017-5022.

[7] D. Bharadia et al., "Full Duplex Radios," in ACM SIGCOMM, 2013, pp. $375-386$.

[8] E.-S. Jung and N. H. Vaidya, "A Power Control MAC Protocol for Ad hoc Networks," in ACM MobiCom, 2002, pp. 36-47.

[9] I. . W. Group et al., "Wireless LAN Medium Access Control (MAC) and Physical Layer (PHY) Specifications," 1997.

[10] G. Bianchi, "Performance analysis of the IEEE 802.11 distributed coordination function," IEEE J. Sel. Areas Commun., vol. 18, no. 3, pp. $535-547,2000$

[11] G. Alfano et al., "New insights into the stochastic geometry analysis of dense csma networks," in IEEE INFOCOM, 2011, pp. 2642-2650.

[12] D. Moltchanov, "Distance distributions in random networks," Ad Hoc Networks, vol. 10, no. 6, pp. 1146-1166, 2012.

[13] T. Bourgeois and S. Shimamoto, "Stochastic analysis of random ad hoc networks with maximum entropy deployments," IJWMC, vol. 6, no. 3 , p. 19,2014 